Animal Reproduction Research Institute,

Agricultural Research Centre, El-Haram, Giza, Egypt.

\title{
STUDIES ON THE EFFECT OF ADDITION OF KOREAN RED GINSENG® AQUEOUS EXTRACTS ON THE FERTILIZING CAPACITY OF RABBIT SEMEN \\ (With 6 Tables)
}

\author{
By \\ ROWIDA M. RIAD; T.S.T. SELEEM* \\ and DALAL S. MOHAMED
}

* Animal Production Research Institute, Agricultural Research Centre, Dokki, Giza, Egypt

(Received at 10/9/2009)

دراسة تأثير إضافة المستخلص المائي لتبات الجنسنج على الكفاءة الإخصابية للسائل المنوي للأرانب لئب المين

رويدا محد رياض ، طارق سلييان توفيق سليي ، دلال سعد اللين محمد

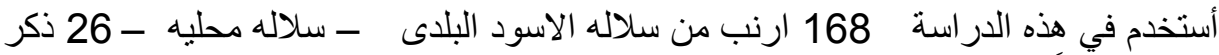

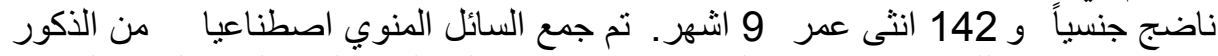

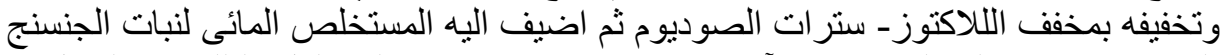

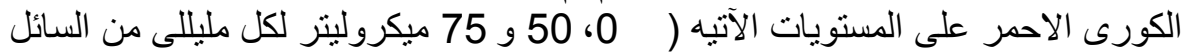

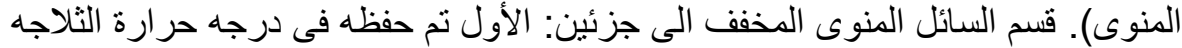

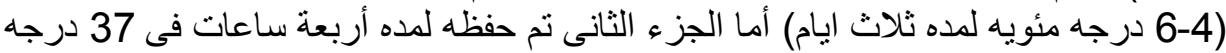

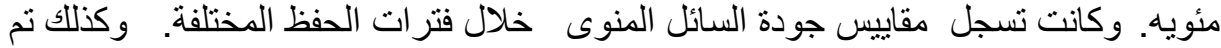

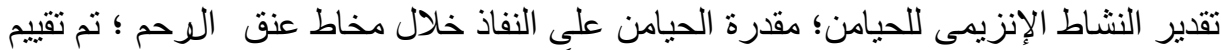

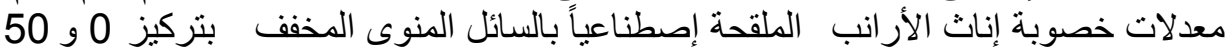

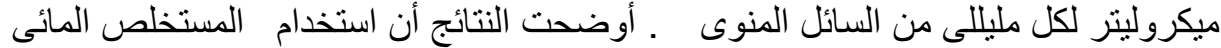

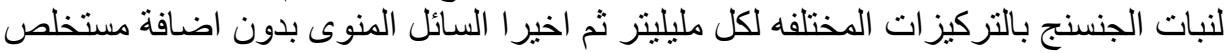

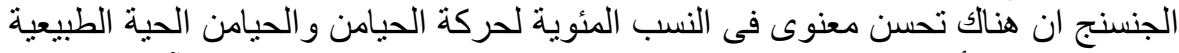

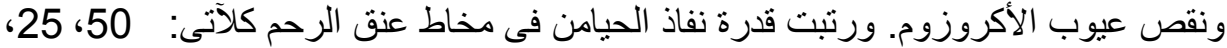

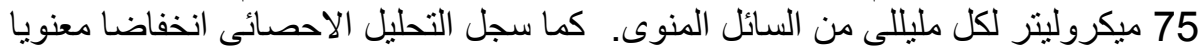

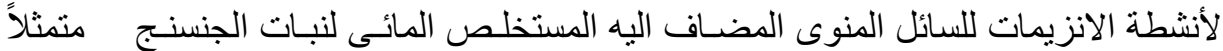

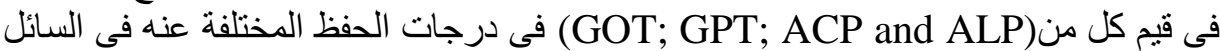

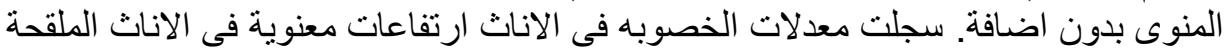

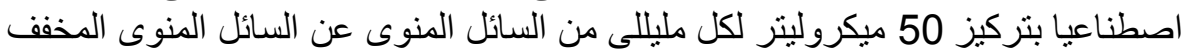


One hundred sixty eight sexually mature Baladi Black (BB) rabbits (26 bucks aged 9 months and 142 monoparous does in two sequence parities) were used in the present study. Semen was collected artificially, pooled and diluted with sodium citrate diluent. Aqueous extracts of Korean red ginseng $®$ were added to the diluted semen at different concentrations of 0 (control), 25, 50, and $75 \mu \mathrm{l} / \mathrm{ml}$, then the diluted semen was divided into two parts, the first part was stored at refrigeration temperature $\left(4-6{ }^{\circ} \mathrm{C}\right)$ for up to 3 days while the second part was incubated at $37{ }^{\circ} \mathrm{C}$ for up to $4 \mathrm{hrs}$. Percentages of sperm motility; alive and normal spermatozoa and acrosomal defects were recorded at the different stages of the preservation. Sperm penetration into estrous cow cervical mucus was estimated during diluted semen incubation. Evaluation of the activities of glutamic-oxaloacetic transaminase (GOT); glutamic -pyruvic transaminase (GPT); acid phosphatase (ACP) and alkaline phosphatase (ALP) enzymes in the BB rabbit semen were measured during preservation. One hundred and forty two rabbit does in two consecutive parities were artificially inseminated using either the diluted semen free or supplemented with $50 \mu$ Korean red ginseng extract/ $1 \mathrm{ml}$ diluted semen (the level which showed the ideal effects on semen quality) to estimate the fertility traits. In both parts of the supplemented diluted rabbit semen with Korean red ginseng ${ }^{\circledR}$ extract showed that there was significant improvement in: $(\mathrm{P} \leq 0.05)$ sperm motility, the percentages of alive spermatozoa, normal spermatozoa and storageability, while the acrosomal defects percentages were significantly $(\mathrm{P} \leq 0.05)$ decreased. Sperm penetration into estrous cow cervical mucus were arranged significantly $(\mathrm{P} \leq 0.05)$ in descending order as obtained by supplemented diluted semen with Korean red ginseng® extract $(50,75,25 \mu \mathrm{l} / \mathrm{ml}$ and the control ones respectively). Also there were significant $(\mathrm{P} \leq 0.05)$ decreases in the mean values of each of GOT, GPT, ACP and ALP either during preservation at refrigeration conditions or at incubation temperature. Conception rate, Kindling rate and litter size values were significantly $(\mathrm{P} \leq 0.05)$ better in rabbit does inseminated artificially by using diluted semen supplemented with $50 \mu \mathrm{l} / \mathrm{ml}$ aqueous extracts of Korean red ginseng ${ }^{\circledR}$ than those inseminated using extended semen free- Korean red ginseng extract. In conclusion, addition of Korean red ginseng $®$ on diluted rabbit semen might improve the semen quality during preservation at different temperatures and fertility traits of rabbit does inseminated artificially. It 
was found that the ideal level was the addition of Korean red ginseng aqueous extracts with $50 \mu \mathrm{l} / 1 \mathrm{ml}$ diluted semen.

Key words: Korean red ginseng®, semen preservation, enzymes, A.I, fertility.

\section{INTRODUCTION}

Ginseng and ginseng products are increasing in popularity. They have been highly valued for thousands of years in many different cultures for their medicinal properties. Korean or Asian, Chinese ginseng known as Panax ginseng is the type most often studied and the most abundant (Tenney, 1996). It is a medicinal herb widely used in Asian countries. Many of its pharmacological actions are attributed to what is commonly called ginsenosides (saponin triterpenoid glycosides) (Minyoung et al., 2007). Some biologically effective components of this medicinal herb possess antioxidative /free radical scavenging properties have been reported to improve sperm functions in vitro and in vivo (Rees et al., 1990; Chen, 1996; Zheng and Zhang, 1997; Suzuki et al., 2003; Zhang et al., 2006). The capacity of sperm fertilization is principally dependent on sperm motility and sperm membrane integrity; fertilization will be impaired if they are damaged. Nitric oxide (NO) is a biologically active free radical and also an important intracellular and intercellular messenger which is generated in mammalian cells from L-arginine by family of nitric oxide synthasas (NOS) (Marletta, 1993). $\mathrm{NO}$ is beneficial to sperm motility as it was indicated to play a significant role in modulation of sperm functions (Lewis et al., 1996) and acrosomal reaction (Revelli et al., 1999). Recently, Ginsenosides have been shown to increase human sperm motility in vitro (Chen et al., 1998, 1999 and 2001and Zhang et al., 2006). Among 30 different Ginsenosides, Ginsenosides Re is the major ingredient of Panax ginseng (Gillis, 1997) and has been widely reported to stimulate the activity of NOS in a variety of cells and tissues (Jin and Liu, 1994; Kang et al., 1995; Jin, 1996; Scott et al., 2001; Bai et al., 2003 and 2004). Zhang et al. (2006) revealed for the first time that Ginsenosides Re improved sperm motility by stimulating a family NOS which enhance the production of Nitric oxide from L-arginine. The aim of the present study, therefore, is to investigate the effects of Korean red ginseng ${ }^{\circledR}$ on the fertilizing ability of rabbit in a trial to improve the semen quality of rabbits for AI. 


\section{MATERIALS and METHODS}

The present work was carried out in an Industrial Rabbitry, near El-Nobareia city, El-Behiera Province, Egypt. A total number of 168 sexually mature Baladi Black (BB) rabbits (26 bucks aged 9 months and 142 monoparous does in two sequence parities) were used in the present study. Korean red ginseng® was purchased from Business Focus Co (produced by Yambyan Betoshan Co., China). At first the study was designed to study the effect of aqueous extracts of Korean red ginseng® at levels $0,25,50$, and $75 \mu \mathrm{l} / \mathrm{ml}$ diluted rabbit semen on its quality, during preservation at different temperatures. Aqueous extracts of Korean red ginseng® were prepared by transfer of 10 grams of the Korean red ginseng ${ }^{\circledR}$ powder to sterile wide-mouthed screw-capped bottles; $250 \mathrm{ml}$ of sterile de-ionized distilled water was added to the powder samples and allowed to be soaked for 3 hours. The mixture was then centrifuged at $1000 \mathrm{rpm}$ for half an hour. The supernatants were filtered through a $0.45 \mu \mathrm{m}$ membrane (Kim et al., 2002). Semen was collected artificially using an artificial vagina as described by Boiti et al. (2005). The ejaculated semen was evaluated microscopically and only ejaculates that showed advanced motility $\geq 70 \%$ were pooled and diluted with glucose yolk citrate diluents and 50000 IU sodium penicillin $+50000 \mu \mathrm{g}$ streptomycin sulphate/ $100 \mathrm{ml}$ diluent at 1: 5 dilution rate. The diluted semen was divided into four portions and supplemented with the different concentrations of Korean red ginseng® extract $(0,25,50$, and $75 \mu \mathrm{l} / \mathrm{ml}$ diluents). Each diluted semen sample was subdivided into two portions, the first was kept at refrigeration condition $\left(4-6{ }^{\circ} \mathrm{C}\right)$ for up to 3 days and the second was incubated at $37{ }^{\circ} \mathrm{C}$ for up to $4 \mathrm{hrs}$. Percentages of advanced sperm motility, alive and morphological normal spermatozoa and acrosomal defects and storageability were recorded at the different stages of preservation according Boiti et al. (2005) and Castellini et al. (2006). After each preservation period, the supplemented extended semen samples were centrifuged at $6000 \mathrm{rpm}$ for 20 minutes before removal of the supernatant and used for enzymatic assay. Activities of glutamic-oxaloacetic transaminase (GOT) and glutamic-pyruvic tranaminase (GPT) enzymes were determined according to Reitman and Frankel (1957) Acid phosphatase (ACP) and alkaline phosphatase (ALP) enzymes were determined calorimetrically according to Graham and Pace (1967). Sperm penetration into estrous cow cervical mucus test was done by using diluted semen supplemented with different levels of Korean red ginseng ${ }^{\circledR}$ extract $(0,25,50$, or $75 \mu 1 /$ 
$\mathrm{ml}$ diluent) and mucus from healthy cows. The mucus was collected at estrous by rubber tube according to (Daader et al., 1989 and Seleem, 1996) and was kept at $-20{ }^{\circ} \mathrm{C}$ until used. The frozen mucus was allowed to thaw at room temperature and then centrifuged at $6000 \mathrm{rpm}$ for 15 minutes. The supernatant was sucked into a $2 \mathrm{~mm}$ internal diameter polythene tube. The mucus was forced inside each tube, from one end. The extended semen was placed into test tubes. The tubes containing mucus were inserted (open end) into the test tubes containing extended semen and incubated at $37{ }^{\circ} \mathrm{C}$ for $4 \mathrm{hrs}$. Sperm mucus penetration was assessed in $\mathrm{mm} / \mathrm{hrs}$ as described by Seleem (1996).

In fertility traits, one hundred forty two BB doe rabbits were divided into two comparable experimental groups (in two sequence parities). BB rabbit does were artificially inseminated with $50 \mu \mathrm{l} / \mathrm{ml}$ Korean red ginseng® -supplemented semen, as this concentration showed improvement in infertility traits than that in the control ones. The artificial insemination was carried out as described by Boiti et al. (2005), conception rate, Kindling rate and litter size were recorded. Animals were fed ad libitum a commercial diet covering the nutritional requirements of the buck and different physiological status of the doe rabbits according to NRC (1977) recommendations. All animals were kept under the same managerial and hygienic conditions and were raised in wired batteries in a windowed rabbitary with natural ventilation. Fresh tab water was automatically available all the time by stainless steel nipples in each cage. Data were subjected to analysis of variance according to Snedecor and Cochran (1982) using the General Linear Model Program of SAS (2001). Percentage values were transformed to Arc. Sin values before being statistically analyzed. Duncan's new multiple range tests was used to test the significance of the differences between means (Duncan, 1955). Number of conceived does and kindling rates were analyzed using the Contingency Tables according to Everitt (1977). 


\section{RESULTS}

Different semen characteristics of BB rabbit bucks supplemented with different concentrations $(25,50$ and $75 \mu \mathrm{l} / \mathrm{ml})$ of Korean red ginseng ${ }^{\circledR}$ extraction during either chilled storage for up to 3 days or at $37^{\circ} \mathrm{C}$ for $4 \mathrm{hrs}$ were shown in Tables (1and 2). From data presented, the overall mean values of the advanced sperm motility percentage, alive spermatozoa percentage, normal spermatozoa percentage and storageability were significantly increased $(\mathrm{P} \leq 0.05)$ in 50 and $75 \mu \mathrm{l} / \mathrm{ml}$ concentrations than that in $25 \mu \mathrm{l} / \mathrm{ml}$ and the control ones. Meanwhile the overall mean values of acrosomal defects decreased significantly $(\mathrm{P} \leq 0.05)$ in 50 and $75 \mu \mathrm{l} / \mathrm{ml}$ concentrations in comparison with that of $25 \mu \mathrm{l} / \mathrm{ml}$ and the control ones.

Korean red ginseng® extract enhanced the sperm penetration into estrous cow cervical mucous. The best concentration for the sperm penetration into estrous cow cervical mucous was $50 \mu \mathrm{l} / \mathrm{ml}$. (Table 3 ). The overall mean values of the different concentrations were significantly increased $(\mathrm{P} \leq 0.05)$ with $50,75,25 \mu \mathrm{l} / \mathrm{ml}$ than that in the control ones respectively.

The result of enzymatic activities in rabbit semen (Tables 4 and 5) showed that the addition of 25, 50 and $75 \mu \mathrm{l} / \mathrm{ml}$ Korean red ginseng ${ }^{\circledR}$ extract on extended $\mathrm{BB}$ rabbit semen significantly $(\mathrm{P} \leq 0.05)$ lowered the release of GOT, GPT, ACP and ALP enzymes into extra cellular medium than that in free red ginseng extraction medium (control) during either chilled storage at $\left(4-6{ }^{\circ} \mathrm{C}\right.$ ) for 3 days or during incubation condition at $37^{\circ} \mathrm{C}$ for $4 \mathrm{hrs}$. It seemed that there were significant differences between the different concentrations of the extract on the enzymatic activity of the spermatozoa, during either semen preservation at refrigeration or incubation conditions. However, the beneficial effects of red ginseng extract on enzymatic activity of spermatozoa were insignificant between the 50 and $75 \mu \mathrm{l} / \mathrm{ml}$ concentrations.

Concerning the fertility traits of the female rabbits, Table (6) showed that there were significantly higher $(\mathrm{P} \leq 0.05)$ conception rates, kindling rates and the litter size at birth in the does artificially inseminated with Red ginseng supplemented diluted semen than that the control one. 
Table 1: Effect of Panax ginseng extraction supplementation with different concentrations on $\mathrm{BB}$ rabbit semen quality during chilled storage at $\left(4-6{ }^{\circ} \mathrm{C}\right)$ for up to three days (Means $\pm \mathrm{SE}$ ).

\begin{tabular}{|c|c|c|c|c|c|}
\hline \multirow{2}{*}{ Items } & \multirow{2}{*}{$\begin{array}{l}\text { Chilled } \\
\text { periods } \\
\text { (Days) }\end{array}$} & \multicolumn{4}{|c|}{ Ginsenosides Re concentration $(\mu \mathrm{l} / \mathrm{ml}$.) } \\
\hline & & control & 25 & 50 & 75 \\
\hline \multirow{3}{*}{$\begin{array}{l}\text { Advanced-sperm } \\
\text { motility }(\%)\end{array}$} & 0.0 & $71.9 \pm 1.9$ & $72.2 \pm 2.2$ & $71.4 \pm 2.6$ & $71.3 \pm 2.1$ \\
\hline & 1.0 & $63.2 \pm 2.2$ & $68.1 \pm 1.7$ & $69.3 \pm 2.4$ & $69.2 \pm 2.3$ \\
\hline & 3.0 & $52.2 \pm 2.0$ & $61.8 \pm 2.0$ & $65.2 \pm 2.7$ & $65.0 \pm 2.4$ \\
\hline \multicolumn{2}{|l|}{ Means $\pm \mathrm{SE}$} & $62.4 \pm 1.4^{\mathrm{c}}$ & $67.4 \pm 1.1^{\mathrm{b}}$ & $68.7 \pm 0.9^{\mathrm{a}}$ & $68.5 \pm 1.2^{\mathrm{a}}$ \\
\hline \multicolumn{2}{|l|}{ Storageability $(\%)^{*}$} & $72.6 \pm 1.4^{\mathrm{c}}$ & $85.6 \pm 1.2^{\mathrm{b}}$ & $91.3 \pm 1.5^{\mathrm{a}}$ & $91.2 \pm 1.3^{\mathrm{a}}$ \\
\hline \multirow{3}{*}{$\begin{array}{l}\text { Alive spermatozoa } \\
(\%)\end{array}$} & 0.0 & $78.1 \pm 2.1$ & $78.6 \pm 2.2$ & $78.5 \pm 2.3$ & $78.3 \pm 2.2$ \\
\hline & 1.0 & $69.7 \pm 2.2$ & $72.1 \pm 2.1$ & $75.1 \pm 2.4$ & $74.6 \pm 2.5$ \\
\hline & 3.0 & $61.2 \pm 1.9$ & $66.1 \pm 2.1$ & $71.0 \pm 2.0$ & $69.7 \pm 2.6$ \\
\hline \multicolumn{2}{|l|}{ Means \pm SE } & $69.7 \pm 0.9^{c}$ & $72.3 \pm 0.9^{\mathrm{b}}$ & $74.9 \pm 1 .^{\mathrm{a}}$ & $74.2 \pm .2^{\mathrm{b}}$ \\
\hline \multirow{3}{*}{$\begin{array}{l}\text { Morphological } \\
\text { normal spermatozoa } \\
(\%)\end{array}$} & 0.0 & $86.1 \pm 2.5$ & $85.8 \pm 2.6$ & $86.0 \pm 2.1$ & $85.7 \pm 2.4$ \\
\hline & 1.0 & $81.8 \pm 2.8$ & $84.3 \pm 2.2$ & $85.1 \pm 2.4$ & $84.8 \pm 2.3$ \\
\hline & 3.0 & $68.9 \pm 2.7$ & $75.6 \pm 2.1$ & $83.7 \pm 2.3$ & $82.9 \pm 2.2$ \\
\hline \multicolumn{2}{|l|}{ Means \pm SE } & $78.9 \pm 1.2^{\mathrm{c}}$ & $81.9 \pm 1.2^{\mathrm{b}}$ & $84.9 \pm 1.4 \mathrm{a}$ & $84.5 \pm 0.8^{\mathrm{a}}$ \\
\hline \multirow{3}{*}{$\begin{array}{l}\text { Acrosomal defects } \\
(\%)\end{array}$} & 0.0 & $13.9 \pm 0.9$ & $14.1 \pm 0.8$ & $13.7 \pm 0.9$ & $13.8 \pm 1.0$ \\
\hline & 1.0 & $17.1 \pm 0.7$ & $16.2 \pm 0.7$ & $15.3 \pm 0.9$ & $15.4 \pm 0.8$ \\
\hline & 3.0 & $19.8 \pm 1.1$ & $18.4 \pm 1.0$ & $17.3 \pm 1.1$ & $17.6 \pm 0.7$ \\
\hline \multicolumn{2}{|l|}{ Means $\pm \mathrm{SE}$} & $16.9 \pm 0.8^{\mathrm{a}}$ & $16.2 \pm 0.6^{\mathrm{b}}$ & $15.4 \pm 0.9^{b}$ & $15.6 \pm 0.6^{b}$ \\
\hline
\end{tabular}

Means within the same row (a, b, c\& d) with different letter superscripts are significantly different at $(\mathrm{P} \leq 0.05)$

Final advanced sperm motility(after 3 days)

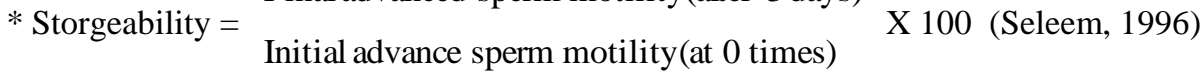


Table 2: Effect of Panax ginseng extraction supplementation with different concentrations on $\mathrm{BB}$ rabbit semen quality during incubation at $37^{\circ} \mathrm{C}$ for up to four hours (Means \pm SE).

\begin{tabular}{|c|c|c|c|c|c|}
\hline \multirow[t]{2}{*}{ Items } & \multirow{2}{*}{$\begin{array}{l}\text { Incubation } \\
\text { periods (Hours) }\end{array}$} & \multicolumn{4}{|c|}{ Ginsenosides Re concentration $(\mu \mathrm{l} / \mathrm{ml}$.) } \\
\hline & & 0 & 25 & 50 & 75 \\
\hline \multirow{4}{*}{$\begin{array}{l}\text { Advanced- } \\
\text { sperm motility } \\
(\%)\end{array}$} & 0.0 & $72.1 \pm 1.7$ & $72.0 \pm 1.8$ & $71.9 \pm 2.2$ & $72.3 \pm 1.9$ \\
\hline & 2.0 & $65.6 \pm .1 .9$ & $68.2 \pm 1.9$ & $69.5 \pm 1.7$ & $69.1 \pm 2.2$ \\
\hline & 4.0 & $58.2 \pm 1.6$ & $63.3 \pm 1.7$ & $66.6 \pm 2.0$ & $66.2 \pm 1.8$ \\
\hline & Means \pm SE & $65.3 \pm 1.1^{\mathrm{c}}$ & $67.8 \pm 1.1^{\mathrm{b}}$ & $69.3 \pm 1.3^{\mathrm{a}}$ & $69.2 \pm 1.2^{\mathrm{a}}$ \\
\hline \multicolumn{2}{|c|}{ Storageability $(\%)^{*}$} & $80.7 \pm 0.8^{\mathrm{c}}$ & $87.9 \pm 1.0^{\mathrm{b}}$ & $92.6 \pm 0.9^{\mathrm{a}}$ & $91.6 \pm 0.8^{\mathrm{a}}$ \\
\hline \multirow{4}{*}{$\begin{array}{l}\text { Alive } \\
\text { spermatozoa }(\%)\end{array}$} & 0.0 & $79.2 \pm 2.2$ & $78.8 \pm 2.2$ & $78.9 \pm 2.2$ & $79.0 \pm 1.7$ \\
\hline & 2.0 & $69.2 \pm 1.8$ & $73.4 \pm 2.2$ & $76.1 \pm 1.2$ & $75.6 \pm 1.3$ \\
\hline & 4.0 & $63.7 \pm 2.2$ & $68.2 \pm 1.9$ & $72.6 \pm 0.8$ & $72.5 \pm 1.0$ \\
\hline & Means \pm SE & $70.7 \pm 1.2^{\mathrm{c}}$ & $73.5 \pm 1.2^{\mathrm{b}}$ & $75.9 \pm 0.9^{\mathrm{a}}$ & $75.7 \pm 1.2^{\mathrm{a}}$ \\
\hline \multirow{4}{*}{$\begin{array}{l}\text { Morphological } \\
\text { normal } \\
\text { spermatozoa }(\%)\end{array}$} & 0.0 & $86.9 \pm 2.1$ & $87.0 \pm 2.3$ & $86.3 \pm 2.2$ & $86.4 \pm 2.4$ \\
\hline & 2.0 & $84.3 \pm 2.5$ & $85.1 \pm 1.9$ & $85.4 \pm 1.7$ & $85.3 \pm 2.0$ \\
\hline & 4.0 & $72.9 \pm 1.7$ & $79.7 \pm 1.9$ & $83.8 \pm 2.1$ & $84.7 \pm 1.6$ \\
\hline & Means \pm SE & $81.4 \pm 1.2^{\mathrm{c}}$ & $83.9 \pm 1.2^{\mathrm{b}}$ & $85.2 \pm 0.8^{\mathrm{a}}$ & $85.5 \pm 1.1^{\mathrm{a}}$ \\
\hline \multirow{4}{*}{$\begin{array}{l}\text { Acrosomal } \\
\text { defects }(\%)\end{array}$} & 0.0 & $13.7 \pm 1.7$ & $13.9 \pm 1.4$ & $14.0 \pm 1.3$ & $14.0 \pm 1.6$ \\
\hline & 2.0 & $16.8 \pm 0.8$ & $15.2 \pm 0.9$ & $14.7 \pm 0.6$ & $14.1 \pm 1.3$ \\
\hline & 4.0 & $18.7 \pm 1.3$ & $17.9 \pm 0.8$ & $16.4 \pm 0.6$ & $17.0 \pm 1.1$ \\
\hline & Means \pm SE & $16.4 \pm 0.4^{\mathrm{a}}$ & $15.7 \pm 0.3^{\mathrm{b}}$ & $15.1 \pm 0.4^{\mathrm{b}}$ & $15.1 \pm 0.6^{\mathrm{b}}$ \\
\hline
\end{tabular}

Means within the same row $(a, b, c \& d)$ with different letter superscripts are significantly different at $(\mathrm{P} \leq 0.05)$

* Storgeability $=\frac{\text { Final advanced sperm motility(after 3 days) }}{\text { Initial advance sperm motility(at } 0 \text { times) }}$ X 100 (Seleem, 1996)

Table 3: Effect of Panax ginseng extraction supplementation with different concentrations on Baladi Black rabbit sperm penetration into estrous cow cervical mucus $(\mathrm{mm} / \mathrm{hr}$.) during incubation at $37^{\circ} \mathrm{C}$ for up to 4 hours.

\begin{tabular}{|c|c|c|c|c|}
\hline \multirow{2}{*}{$\begin{array}{c}\text { Incubation } \\
\text { periods } \\
\text { (Hours) }\end{array}$} & \multicolumn{4}{|c|}{ Ginsenosides Re concentration $(\mu \mathrm{l} / \mathrm{ml})}$. \\
\cline { 2 - 5 } & Control & 25 & 50 & 75 \\
\hline 1 & $17.4 \pm 2.2$ & $18.3 \pm 2.4$ & $34.1 \pm 2.7$ & $28.4 \pm 2.4$ \\
\hline 2 & $30.8 \pm 3.1$ & $36.3 \pm 2.9$ & $52.1 \pm 3.3$ & $46.9 \pm 2.4$ \\
\hline 3 & $43.2 \pm 3.6$ & $54.9 \pm 3.2$ & $79.3 \pm 3.4$ & $62.1 \pm 3.5$ \\
\hline 4 & $64.1 \pm 3.6$ & $69.5 \pm 3.7$ & $93.2 \pm 3.9$ & $79.4 \pm 3.7$ \\
\hline Means \pm SE & $38.9 \pm 2.1^{\mathrm{d}}$ & $44.8 \pm 3.1^{\mathrm{c}}$ & $64.7 \pm 3.1^{\mathrm{a}}$ & $54.2 \pm 3.4^{\mathrm{b}}$ \\
\hline
\end{tabular}

Means within the same row (a, b, c\& d) with different letter superscripts are significantly different at $(\mathrm{P} \leq 0.05)$ 
Table 4: Effect of Panax ginseng supplementation with different concentrations on the activities of GOT, GPT, ACP and ALP enzymes in the diluted $\mathrm{BB}$ rabbit semen during chilled storage at $\left(4-6{ }^{\circ} \mathrm{C}\right)$ for up to 3 days.

\begin{tabular}{|c|c|c|c|c|c|}
\hline \multirow{2}{*}{ Items } & \multirow{2}{*}{$\begin{array}{l}\text { Chilled } \\
\text { periods } \\
\text { (Days) }\end{array}$} & \multicolumn{4}{|c|}{ Ginsenosides Re concentration $(\mu \mathrm{l} / \mathrm{ml}$.) } \\
\hline & & 0 & 25 & 50 & 75 \\
\hline \multirow{3}{*}{$\begin{array}{l}\text { GOT } \\
(\mathrm{U} / \mathrm{L})\end{array}$} & 0.0 & $22.7 \pm 0.9$ & $23.0 \pm 1.0$ & $23.2 \pm 0.8$ & $23.1 \pm 0.9$ \\
\hline & 1.0 & $31.3 \pm 1.3$ & $28.6 \pm 1.1$ & $24.8 \pm 1.2$ & $24.0 \pm 1.0$ \\
\hline & 3.0 & $39.1 \pm 1.3$ & $35.2 \pm 1.3$ & $30.1 \pm 0.9$ & $30.0 \pm 1.1$ \\
\hline \multicolumn{2}{|c|}{ Means \pm SE } & $31.1 \pm 0.9^{\mathrm{a}}$ & $28.9 \pm 0.8^{b}$ & $26.1 \pm 0.7^{c}$ & $25.7 \pm 0.4^{c}$ \\
\hline \multirow{3}{*}{$\begin{array}{l}\text { GPT } \\
(\mathrm{U} / \mathrm{L})\end{array}$} & 0.0 & $10.5 \pm 0.5$ & $10.7 \pm 0.5$ & $10.4 \pm 0.9$ & $10.8 \pm 0.8$ \\
\hline & 1.0 & $16.8 \pm 1.0$ & $14.6 \pm 0.9$ & $13.3 \pm 1.4$ & $13.0 \pm 13$ \\
\hline & 3.0 & $24.9 \pm 1.1$ & $19.2 \pm 1.3$ & $16.2 \pm 1.4$ & $15.4 \pm 1.4$ \\
\hline \multicolumn{2}{|c|}{ Means \pm SE } & $17.4 \pm 0.9^{\mathrm{a}}$ & $14.8 \pm 0.6^{b}$ & $13.3 \pm 0.5^{c}$ & $13.1 \pm 0.7^{\mathrm{c}}$ \\
\hline \multirow{3}{*}{$\begin{array}{c}\text { ACP } \\
\text { (U/106sp.) }\end{array}$} & 0.0 & $11.1 \pm 0.7$ & $11.5 \pm 0.7$ & $11.2 \pm 0.8$ & $11.5 \pm 0.7$ \\
\hline & 1.0 & $17.3 \pm 0.9$ & $15.5 \pm 0.8$ & $13.4 \pm 0.8$ & $13.1 \pm 0.8$ \\
\hline & 3.0 & $22.1 \pm 1.2$ & $17.9 \pm 1.2$ & $17.0 \pm 1.1$ & $16.7 \pm 1.2$ \\
\hline \multicolumn{2}{|c|}{ Means \pm SE } & $16.8 \pm 0.8^{a}$ & $15.0 \pm 0.5^{b}$ & $13.9 \pm 0.5^{c}$ & $13.8 \pm 0.6^{c}$ \\
\hline \multirow{3}{*}{$\begin{array}{c}\text { ALP } \\
\text { (U/106sp.) }\end{array}$} & 0.0 & $20.3 \pm 1.0$ & $20.5 \pm 1.3$ & $20.6 \pm 1.1$ & $20.7 \pm 1.2$ \\
\hline & 1.0 & $31.1 \pm 1.5$ & $28.4 \pm 1.2$ & $25.2 \pm 1.6$ & $25.3 \pm 1.4$ \\
\hline & 3.0 & $40.3 \pm 1.9$ & $35.6 \pm 1.4$ & $30.9 \pm 1.6$ & $29.9 \pm 1.5$ \\
\hline \multicolumn{2}{|c|}{ Means \pm SE } & $30.6 \pm 1.0^{\mathrm{a}}$ & $28.2 \pm 0.9^{b}$ & $25.6 \pm 1.1^{\mathrm{c}}$ & $25.3 \pm 1.0^{\mathrm{c}}$ \\
\hline
\end{tabular}

Means within the same row (a, b, c\& d) with different letter superscripts are significantly different at $(\mathrm{P} \leq 0.05)$ 
Table 5: Effect of Panax ginseng supplementation with different concentrations on the activities of GOT, GPT, ACP and ALP enzymes in the diluted $\mathrm{BB}$ rabbit semen during incubation at $37^{\circ} \mathrm{C}$ for up to 4 hours.

\begin{tabular}{|c|c|c|c|c|c|}
\hline \multirow[b]{2}{*}{ Items } & \multirow{2}{*}{$\begin{array}{c}\text { Incubatio } \\
\text { n period } \\
\text { (Hours) }\end{array}$} & \multicolumn{4}{|c|}{ Ginsenosides Re concentration $(\mu \mathrm{l} / \mathrm{ml}$.) } \\
\hline & & 0 & 25 & 50 & 75 \\
\hline \multirow{3}{*}{$\begin{array}{l}\text { GOT } \\
\text { (U/L) }\end{array}$} & 0.0 & $22.1 \pm 0.8$ & $22.5 \pm 0.8$ & $22.8 \pm 0.9$ & $23.1 \pm 0.8$ \\
\hline & 2.0 & $32.3 \pm 1.3$ & $27.6 \pm 1.1$ & $23.6 \pm 1.0$ & $23.4 \pm 1.3$ \\
\hline & 4.0 & $38.7 \pm 1.5$ & $34.8 \pm 1.2$ & $30.3 \pm 1.3$ & $30.3 \pm 1.2$ \\
\hline \multicolumn{2}{|c|}{ Means \pm SE } & $31.0 \pm 1.3^{\mathrm{a}}$ & $28.3 \pm 1.1^{b}$ & $25.6 \pm 0.8^{\mathrm{c}}$ & $25.6 \pm 0.8^{\mathrm{c}}$ \\
\hline \multirow{3}{*}{$\begin{array}{l}\text { GPT } \\
(\mathrm{U} / \mathrm{L})\end{array}$} & 0.0 & $10.4 \pm 0.6$ & $10.5 \pm 0.5$ & $10.5 \pm 0.7$ & $10.6 \pm 0.5$ \\
\hline & 2.0 & $16.2 \pm 0.8$ & $14.1 \pm 0.6$ & $12.9 \pm 0.7$ & $12.7 \pm 0.8$ \\
\hline & 4.0 & $24.1 \pm 1.1$ & $19.3 \pm 1.1$ & $16.8 \pm 1.0$ & $16.4 \pm 1.1$ \\
\hline \multicolumn{2}{|c|}{ Means \pm SE } & $16.9 \pm 0.8^{\mathrm{a}}$ & $14.6 \pm 0.8^{b}$ & $13.4 \pm 0.6^{\mathrm{c}}$ & $13.2 \pm 0.6^{\mathrm{c}}$ \\
\hline \multirow{3}{*}{$\begin{array}{c}\mathrm{ACP} \\
\left(\mathrm{U} / 10^{6} \mathrm{sp} .\right)\end{array}$} & 0.0 & $11.3 \pm 0.7$ & $11.4 \pm 0.6$ & $11.4 \pm 0.7$ & $11.4 \pm 0.5$ \\
\hline & 2.0 & $16.7 \pm 1.1$ & $14.2 \pm 1.2$ & $13.9 \pm 1.1$ & $13.6 \pm 1.0$ \\
\hline & 4.0 & $21.9 \pm 1.2$ & $16.9 \pm 1.4$ & $16.1 \pm 1.2$ & $15.9 \pm 1.1$ \\
\hline \multicolumn{2}{|c|}{ Means \pm SE } & $16.6 \pm 1.1^{\mathrm{a}}$ & $14.2 \pm 0.8^{b}$ & $13.8 \pm 0.7^{\mathrm{c}}$ & $13.6 \pm 0.6^{\mathrm{c}}$ \\
\hline \multirow{3}{*}{$\begin{array}{c}\text { ALP } \\
\left(\mathrm{U} / 10^{6} \mathrm{sp} .\right)\end{array}$} & 0.0 & $20.2 \pm 11$ & $20.5 \pm 1.1$ & $20.5 \pm 1.0$ & $20.7 \pm 1.1$ \\
\hline & 2.0 & $30.9 \pm 1.4$ & $26.7 \pm 1.2$ & $24.2 \pm 1.0$ & $23.4 \pm 1.2$ \\
\hline & 4.0 & $37.7 \pm 1.7$ & $33.2 \pm 1.3$ & $28.0 \pm 1.1$ & $27.9 \pm 1.1$ \\
\hline \multicolumn{2}{|c|}{ Means \pm SE } & $29.6 \pm 1.6^{\mathrm{a}}$ & $26.8 \pm 1.1^{b}$ & $24.2 \pm 0.6^{\mathrm{c}}$ & $24.0 \pm 0.7^{\mathrm{c}}$ \\
\hline
\end{tabular}

Means within the same row ( $\mathrm{a}, \mathrm{b}, \mathrm{c} \& \mathrm{~d}$ ) with different letter superscripts significantly different at $(\mathrm{P} \leq 0.05)$

Table 6: Fertility traits of BB doe rabbits inseminated artificially by using supplemented diluted semen with Panax ginseng extraction.

\begin{tabular}{|l|c|c|}
\hline Items & (Control) & $\begin{array}{c}\text { Panax ginseng levels } \\
(50 \mu \mathrm{l} / \mathrm{ml} .)\end{array}$ \\
\hline No. of mated does & $71 \times 2^{*}$ & $71 \times 2^{*}$ \\
\hline No. of conceived does & $90^{\mathrm{b}}(63.38 \%)$ & $117^{\mathrm{a}}(82.4 \%)$ \\
\hline Kindling rate $(\%)$ & $63.38^{\mathrm{b}}$ & $82.39^{\mathrm{a}}$ \\
\hline Litter size at birth & $6.72 \pm 0.8^{\mathrm{b}}$ & $7.91 \pm 0.9^{\mathrm{a}}$ \\
\hline
\end{tabular}

Means within the same row (a \& b) with different letter superscripts are significantly different at $(\mathrm{P} \leq 0.05)$.

* $71 \mathrm{X} 2$ means 71 does were inseminated in two sequence parities 


\section{DISCUSSION}

The present study revealed that Korean red ginseng® improved the different semen characteristics of $\mathrm{BB}$ rabbit (sperm motility, the percentages of alive spermatozoa, normal spermatozoa and storageability were increased, while the acrosomal defects percentages were decreased). These results were in agreement with those of Hellstrom et al. (1994); Zhang and Zheng, (1996); Zini et al. (1995); Herrero et al. (1999); Yeoman et al. (1998); Sengoku et al. (1998) and Zhang et al. (2006), who confirmed that Ginsenosides Re improved human sperm motility, also improved the survival rate and sperm quality in guinea pigs (Hwang et al., 2004) as well as Panax ginseng might have applications in the recovery of male mice infertility arising from azoospermia and oligospermia (Minyoung et al., 2007). The stimulating function of Ginseng to NO be beneficial to sperm motility as it was indicated to play a significant role in modulation of sperm functions (Lewis et al., 1996) and acrosomal reaction (Revelli 1999). It was proved that Ginsenosides Re have a role in stimulating a family NOS and enhance the production of $\mathrm{NO}$ which can be generated by spermatozoa themselves, and both endogenous and exogenous NO play an important role in improvement of sperm functions (Lewis et al., 1996; Revelli et al., 1999; Hellstrom et al., 1994; Zhang and Zheng, 1996).

The present study revealed that the concentration of $50 \mu \mathrm{l} / \mathrm{ml}$ was more effective than the concentration of $75 \mu \mathrm{l} / \mathrm{ml}$ for the sperm penetration into estrous cow cervical mucous. Ginsenosides Re exerts its role of increasing sperm motility is involved partially by its property as a free radical scavenger (Chen, 1996; Chu and Chen, 1990). The effects of NO on sperm functions are biphasic: at lower concentrations, NO improves sperm functions (Hellstrom et al., 1994; Zhang and Zheng, 1996; Zini et al., 1995; Herrero et al., 1999; Yeoman et al., 1998; Sengoku et al., 1998); and the high concentration of NO has a deleterious effect on sperm motility (Zhang and Zheng, 1996; Zini et al., 1995; Tomlinson et al., 1992; Weinberg et al., 1995).

Korean red ginseng® extract lowered the release of GOT, GPT, ACP and ALP enzymes into the extra cellular medium. It is known that the capacity of the sperm fertilization is principally dependent on sperm motility and sperm membrane integrity; fertilization will be impaired if they are damaged (Zhang et al., 2006). The effects of ROS (reactive oxygen species such as superoxide anion (O2.) and hydrogen peroxide) 
which are generated by the abnormal spermatozoa to sperm plasma membrane are thought to be responsible for loss of sperm motility and the other sperm functions (Alvarez et al., 1987; Aitken and Clarkson, 1987; Iwasaki and Gagnon, 1992; Jone et al., 1978). Ginseng was proved to act as exogenous antioxidants/ ROS scavengers (Rees et al., 1990; Zheng and Zhang, 1997; Suzuki et al., 2003; Keskes-Ammar et al., 2003). Consequently the sperm functions can be improved with ginseng supplementation.

Concerning the fertility traits, there were non-significant changes between 50 and $75 \mu \mathrm{l} / \mathrm{ml}$ in all results except that in the $50 \mu \mathrm{l} / \mathrm{ml}$ concentration revealed significant increase in alive spermatozoa \% during chilled storage at $4-6{ }^{\circ} \mathrm{C}$ and mucous penetration, so does were artificially inseminated by diluted pooled semen supplemented with 50 $\mu 1 / \mathrm{ml}$. The present result showed an improvement in semen quality and inhibiting the sperm enzymatic breakdown, this result agreed with those of (Hellstrom et al., 1994; Zhang and Zheng, 1996; Zini et al., 1995; Herrero et al., 1999; Yeoman et al., 1998; Sengoku et al., 1998 and Zhang et al., 2006) who shown that exogenous NO donors have indeed been to enhance sperm motility in vitro, viability, hyperactivation, capacitation and sperm binding to the zona pellucida. Finally the aqueous extract of ginseng are composed of mixture of glycosides, ginsonsides, trace minerals (calcium, iron, phosphorus, sodium, silicon, potassium, manganese, magnesium, and sulphur), vitamins (vitamin A, vitamin E, Bcomplex: thiamin, riboflavin, B12, and niacin (Kim et al., 2002; Park, 1969) and as a result it improves the diluent of rabbit semen.

Our results suggest that aqueous extract of Korean red ginseng® supplemented semen is beneficial and improve diluted semen quality of rabbit, and we can recommend the artificial insemination of doe rabbits with Korean red ginseng ${ }^{\circledR}$ supplemented buck semen to improve the does' fertility traits. Also further investigations are need for studying the effect of ginseng in reproduction of large animals.

\section{REFERENCES}

Aitken, R.J. and Clarkson, J.S. (1987): Cellular basis of defective sperm function and its association with the genesis of reactive oxygen species by human spermatozoa. J. Reprod. Fertil., 81: 459-469. Alvarez, J.G.; Touchstone, J.C.; Blasco, L. and Storey, B.T. (1987): Spontaneous lipid peroxidation and production of hydrogen Spontaneous lipid peroxidation and production of hydrogen 
peroxide and superoxide in human spermatozoa. Superoxidedismutase as major enzyme protectant against oxygen toxicity. J. Androl., 8: 338-348.

Bai, C.X.; Sunami, A.; Namiki, T.; Sawanobori, T. and Furukawa, T. (2003): Electrophysiological effects of ginseng and 150 Hong Zhang et al. ginsenoside $\mathrm{Re}$ in guinea pig ventricular myocytes. Eur. J. Pharmacol., 476: 35-44.

Bai, C.X.; Takahashi, K.; Masumiya, H.; Sawanobori, T. and Furukawa, T. (2004): Nitric oxide-dependent modulation of the delayed rectifier $\mathrm{K}+$ current and the L-type $\mathrm{Ca} 2+$ current by ginsenoside Re, an ingredient of Panax ginseng, in guineapig cardiomyocytes. Br. J. Pharmacol., 142: 567-575.

Boiti, C.; Castellini, M.; Thau-Clément, M.; Besenfelder, U.; Liguori, L.; Renieri, T. and Pizzi, F. (2005): Guidelines for the handling of rabbit bucks and semen. World Rabbit Science, 13: 71-91.

Castellini, C.; Besenfelder, U.; Pizzi, F.; Theau-Clément, M.; Vicente, J.S.A. and Renieri, T. (2006): Recent Knowledge on Rabbit Semen and Buck Management. (EU- Cost) Ed. Maertens L., Coudert pp. 53-67.

Chen, J.C.; Chen, L.D.; Tsauer, W.; Tsai, C.C.; Chen, B.C. and Chen, Y.J. (2001): Effects of Ginsenoside Rb2 and Rc on inferior human sperm motility in vitro. Am. J. Chin. Med., 29: 155-160.

Chen, J.C.; Xu, M.X.; Chen, L.D.; Chen, Y.N. and Chiu, T.H. (1998): Effect of panax notoginseng saponins on sperm motility and progression in vitro. Phytomedicin, 5: 289-292.

Chen, J.C.; Xu, M.X.; Chen, L.D.; Chen, Y.N. and Chiu, T.H. (1999): Effect of panax notoginseng extracts on inferior sperm motility in vitro. Am. J. Chin. Med., 27: 123-128.

Chen, X. (1996): Cardiovascular protection by ginsenosides and their nitric oxide releasing action. Clin. Exp. Pharmacol. Physiol., 23: 728-732.

Chu, G.X. and Chen, X. (1990): Anti-lipid peroxidation and protection of ginsenosides against ischemia and reperfusion injuries in rats. Acta Pharmacol. Sin., 11: 119-123.

Daader, A.H.; El-Gaafary, M.N. and Zeidan, A.E.B. (1989): Stimulation of bull sperm during chilled storage by using caffeine. 3rd Egyptian-British Conference on Animal Fish and Poultry Production, Alexandria, pp. 672.

Duncan, D.B. (1955): Multiple Range and Multiple (F-test). Biometrics, 11: $1-42$. 
Everitt, B.S. (1977): The Analysis of Contingency Tables. Monographs of Applied Probability and Statistics. pp, 38-66 London, Chopman Hall.

Gillis, C.N. (1997): Panax ginseng pharmacology: a nitric oxide link? Biochem. Pharmacol., 54: 1-8

Graham, E.F. and Pace, M.M. (1967): Some biochemical changes in spermatozoa due to freezing. Cryobiology, 4: 75-84.

Hellstrom, W.J.G.; Bell, M.; Wang, R. and Sikka, S.C. (1994): Effect of sodium nitroprusside on sperm motility, viability and lipid peroxidation. Fertil. Steril., 61: 1117-1122.

Herrero, M.B.; De Lamirande, E. and Gagnon, C. (1999): Nitric oxide regulates human sperm capacitation and protein-tyrosine phosphorylation in vitro. Biol. Reprod., 61: 575-581.

Hwang Seock-Yeon, S.Y.; Wun-Jae Kim; Jae-Joon Wee; Jong-Soon Choi and Si-Kwan Kim (2004): Panax ginseng improves survival and sperm quality in guinea pigs exposed to $2,3,7,8$ tetrachlorodibenzo- p-dioxin. BJU International: 94 (4) pp: 663-668.

Iwasaki, A. and Gagnon, C. (1992): Formation of reactive oxygen species in spermatozoa of infertile patients. Fertil. Steril., 57: 409-416.

Jin, Z.Q. and Liu, C.M. (1994): Effect of ginsenoside Re on the electrophysiological activity of the heart. Planta Med., 60: 192-193.

Jin, Z.Q. (1996): The action of ginsenoside Re on inotropy and chronotropy of isolated atria prepared from guinea pigs. Planta Med., 62: 314-316.

Jone, R.; Mann, T. and Sherins, R. (1978): Adverse effects of peroxidized lipid on human spermatozoa. Proc. R. Soc. Lond., 201: 413-417.

Kang, S.Y.; Schini-Kerth, V.B. and Kim, N.D. (1995): Ginsenosides of the protopanaxatriol group cause endothelium-dependent relaxation in the rat aorta. Life Sci., 56: 1577-1586.

Keskes-Ammar, L.; Feki-Chakroun, N.; Rebai, T.; Sahnoun, Z.; Ghozzi, H.; Hammami, S.; Zghal, K.; Fki, H.; Damak, J. and Bahloul, A. (2003): Sperm oxidative stress and the effect of an oral vitamin $\mathrm{E}$ and selenium supplement on semen quality in infertile men. Arch. Androl., 49: 83-94. 
Kim, Y.K.; Guo, Q. and Packer, L. (2002): Free radical scavenging activity of red gensing aqueous extracts. Toxicology, 172: pp. 149-156.

Lewis, S.E.; Donnelly, E.T.; Sterling, E.S.; Kennedy, M.S.; Thompson, W. and Chakravarthy, U. (1996): Nitric oxide synthase and nitrite production in human spermatozoa: evidence that endogenous nitric oxide is beneficial to sperm motility. Mol. Hum. Reprod., 2: 873-878.

Marletta, M.A. (1993): Nitric oxide synthase structure and mechanism. J. Biol. Chem., 268: 12231-12234.

Minyoung, J.I.; Naogiro Minami; Masayasu Yamada and Hirishi Imai (2007): Effect of protopanaxatriol saponin on spermatogenic stem cell survival in busulfan-treated male mice. Repro. Med. and Biol. 6: 99-108.

NRC (1977): National Research Council: Nutrient Requirements of Rabbits. $2^{\text {nd }}$ Revised Edition, National Academy of Sciences, Washington, DC. USA.

Park, J.D. (1969): Recent studies on the chemical consitutes of Korean ginsing (Panax ginseng C.A. Meyer). Kor. J. Ginseing Sci. 20: 389-415.

Rees, J.M.; Ford, W.C. and Hull, M.G. (1990): Effect of caffeine and of pentoxifylline on the motility and metabolism of human spermatozoa. J. Reprod. Fertil., 90: 147-156.

Reitman, S. and Frankel, S. (1957): Colorimetric methods for the determination of serum glutamic oxaloacetic and glutamic pyruvic traminases. Amer. J. Clin. Path, 2: pp. 56.

Revelli, A.; Soldati, G.; Costamagna, C.; Pellerey, O.; Aldieri, E.; Massobrio, M.; Bosia, A. and Ghigo, D. (1999): Follicular fluid proteins stimulate nitric oxide (NO) synthesis in human sperm: a possible role for NO in acrosomal reaction. J. Cell Physiol., 178: 85-92.

SAS (2001): 0 SAS/ STAT User's Guide. (Version $6,4^{\text {th }}$ ed.). SAS Inst. Inc., Cary, NC.

Scott, G.I.; Colligan, P.B.; Ren, B.H. and Ren, J. (2001): Ginsenosides $\mathrm{Rb} 1$ and $\mathrm{Re}$ decrease cardiac contraction in adult rat ventricular myocytes: role of nitric oxide. Br. J. Pharmacol., 134: 1159-1165.

Seleem, T.S.T. (1996): Studies on some reproductive characteristics in rabbits. M. Science, Thesis, Faculty Agriculture, Zagazig University, Zagazig, Egypt. 
Sengoku, K.; Tamate, K.; Yoshida, T.; Takaoka, Y.; Miyamoto, T. and Ishikaw, M. (1998): Effects of low concentrations of nitric oxide on the zona pellucida binding ability of human spermatozoa. Fertil. Steril., 69: 522-527.

Snedecor, G.W. and Cochran, W.G. (1982): Statistical Methods. $2^{\text {nd }}$ Ed. Iowa Univ. Press. Ames, Iowa.

Suzuki, M.; Kurabayashi, T.; Yamamoto, Y.; Fujita, K. and Tanaka, K. (2003): Effects of antioxidant treatment in oligozoospermic and asthenozoospermic men. J. Reprod. Med., 48: 707-712.

Tenney, D. (1996): Ginseng. Woodland Publishing, Inc. P.O. Box 160 Pleasant Grove, UT 84062 pp. 5.

Tomlinson, M.J.; East, S.J.; Barratt, C.L.; Bolton, C.L. and Cooke, I.D. (1992): Possible role of reactive nitrogen intermediates in leukocyte-mediated sperm dysfunction. Am. J. Reprod. Immunol., 27: 89-92.

Weinberg, J.B.; Doty, E.; Bonaventura, J. and Haney, A.F. (1995): Nitric oxide inhibition of human sperm motility. Fertil. Steril., 64: 408-413.

Yeoman, R.R.; Jones, W.D. and Rizk, B.M. (1998): Evidence for nitric oxide regulation of hamster sperm hyperactivation. J. Androl., 19: 58-64.

Zhang, H. and Zheng, R.L. (1996): Possible role of nitric oxide on fertile and asthenozoospermic infertile human sperm functions. Free Radic. Res., 25: 347-354.

Zhang; Qing-Ming Zhou; Xiao-Da Li; Yi Xie; Xin Duan; Feng-Ling Min; Bing Liu, and Zhi Gang Yuan (2006): Ginsenoside Re Increases Fertile and Asthenozoospermic Infertile Human Sperm Motility by Induction of Nitric Oxide Synthase. Arch. Pharm. Res. 29 (2): 145-151.

Zheng, R.L. and Zhang, H. (1997): Effects of ferulic acid on fertile and asthenozoospermic infertile human sperm motility, viability, lipid peroxidation, and cyclic nucleotides. Free Radic. Biol. Med.: 22: 581-586.

Zini, A.; De Lamirande, E. and Gagnon, C. (1995): Low levels of nitric oxide promote human sperm capacitation in vitro. J. Androl., 16: 424-430. 